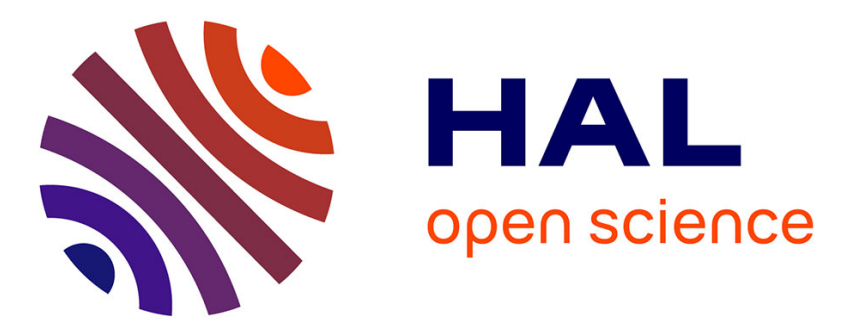

\title{
Coordinated Path Following Control of Multiple Wheeled Mobile Robots Through Decentralized Speed Adaptation
}

Xianbo Xiang, Lionel Lapierre, Bruno Jouvencel, Olivier Parodi

\section{- To cite this version:}

Xianbo Xiang, Lionel Lapierre, Bruno Jouvencel, Olivier Parodi. Coordinated Path Following Control of Multiple Wheeled Mobile Robots Through Decentralized Speed Adaptation. IROS: Intelligent Robots and Systems, Oct 2009, St Louis, MO, United States. pp.4547-4552. hal-00733800

\section{HAL Id: hal-00733800 https://hal.science/hal-00733800}

Submitted on 19 Sep 2012

HAL is a multi-disciplinary open access archive for the deposit and dissemination of scientific research documents, whether they are published or not. The documents may come from teaching and research institutions in France or abroad, or from public or private research centers.
L'archive ouverte pluridisciplinaire HAL, est destinée au dépôt et à la diffusion de documents scientifiques de niveau recherche, publiés ou non, émanant des établissements d'enseignement et de recherche français ou étrangers, des laboratoires publics ou privés. 


\title{
Coordinated Path Following Control of Multiple Wheeled Mobile Robots Through Decentralized Speed Adaptation
}

\author{
Xianbo Xiang, Lionel Lapierre, Bruno Jouvencel and Olivier Parodi
}

\begin{abstract}
This paper addresses the problem of coordinated path following of multiple wheeled mobile robots while keeping a desired formation. The control laws proposed are categorized into two envelopes, one is steering individual robots to trace along predefined paths, and the other is ensuring tracked paths to be well defined in the formation, by means of decentralized speed adaption. Within this framework, geometric paths following are built on Lyapunov theory and backstepping techniques, while injecting helmsman like behavior into individual path following control. Speed adaption with minimum communication variable under the constraints of multi-robot communication topology, is elaborately designed without relative speeds between neighboring robots requested. The simple but effective controller design, enables multi-robot system to be coordinated and stabilized into an invariant manifold, and all speeds converge to desired profiles in addition. Simulation results illustrate the efficacy of the solution proposed.
\end{abstract}

\section{INTRODUCTION}

Nowadays, there is growing interest in the problem of coordinated control of multiple autonomous vehicles. Examples include spacecraft formation flying control [1], cooperative control of mobile robots [2], traffic automated highway systems [3], and coordinated control of underwater vehicles [4]. Control design on multi-vehicle system poses significant theoretical and practical challenges, where coordinated path following is one of them. Other than developing well-done individual path following controller for each vehicle, the counterpart strategy addressing the problem in this paper, is that the dedicated controller dealing with inter-vehicle speed adaptation to keep the coordinated formation, is decentralized. Moreover, the amount of information exchanged between any two vehicles to fulfill the global control requirements, is minimized to one single variable. This single variable proposed here, is the curvilinear abscissa $s_{i}$ (along path length) parameterizing the $i$ th path, and there are no relative speeds between neighboring vehicles requested in the control strategy of speed adaption, which is useful in practical case.

As pointed out in the work of [5], [6], a leader-follower structure for path following control is adopted. However in [5], it requires a large amount of kinematics and dynamics information, be exchanged between leader and follower, besides complex computation of trajectory tracking controllers as a complement of path following controller. In

This work was funded by the EU FreeSubNet project under grant 036186, and the first author was supported by the European Marie Curie Fellowship.

All four authors are with Department of Robotics, CNRS-LIRMMUMII, UMR 5506-CC 477, 161 rue Ada, 34392 Montpellier Cedex 5, France. \{xiang, lapierre, jouvencel and Parodi\}elirmm.fr
[6], an important idea of decoupling the spatial assignment (predefined path) and temporal assignment (desired speed) is proposed. The nonlinear feedback law yields convergence of the two robots to the respective paths and forces the follower to accurately track the leader asymptotically. Moreover, only the path parameter of the leader is required to be sent to the follower, which presents a minimum load in the communication network. Unfortunately, this approach can not be easily generalized to more than two robots. On the other hand, the inherent centralized characteristic of the leader-follower control system, is vulnerable by single-point failure. For instance, any follower who can not keep up with the leader will fail out of the group, and the whole group will collapse if the leader is failed.

A natural way for coordinated control of multiple robots is to built a leaderless strategy [7], and all robots have the same priority to reach the coordinated task. This kind of coordination scheme also exhibits robustness against single robots failures, which means the strategy of decentralized control for coordinating multiple robots is preferred to centralized control scheme. In addition, communication signals required in decentralized system are significantly reduced compared with those in centralized control system. Therefore, decentralized control is much more applicable for realworld communication situation, especially in the situation of severely constrained bandwidth.

Furthermore, information flow among vehicles in the communication network must be carefully treated, which plays a key role in decentralized control of multiple vehicles. In [8], [9], graph is introduced to represent communication network, where each vehicle is one node and each communication link is one edge in the graph. Subsequently, algebraic graph theory supports a rigid methodology to explicitly interpret the relationship between information flow and stability of the cooperating behavior of multiple vehicles. Hopefully, the elegant technique sheds some light into the problem of coordinated path following for multi-vehicle system. In this guidance, local convergence has been resulted by resorting to feedback linearization approach to stabilize a fleet of wheeled robots. The global performance has been obtained for fully actuated underwater vehicles, and error dynamics derived from path parameter is the root to design the coordinated controllers in [10].

In the leading methods reviewed above for coordinated path following control of multiple vehicles, there is a consensus behind the different control techniques in [10] and in [11]. That is, individual path following control and intervehicle coordination are decoupled, so that the essence of 
coordinating task is synchronizing suitable state variable to keep the formation. Moreover, path can be parameterized by the curvilinear path length, which means synchronization can be achieved by adjusting the speed of each vehicles along the path. Therefore, a direct way by regulating speeds of vehicles is intuitive, and this straightforward mechanism is able to simplify the solution, which is inspiring the simple but effective control design in this paper. The decentralized control laws on inter-vehicle speed adaption with minimum communicated variables (i.e. path parameters), enables multivehicle system without all-to-all communications, to be stabilized into an invariant manifold.

This paper is organized as follows. Section II introduces non-singular path following for individual vehicle, with theoretical improvement by injecting helmsman like behavior as a heading reference. Another important contribution, however, is summarized in section III, where a decentralized speed adaptation is designed for coordinated path following of multiple vehicles under communication constraints. The performance of the control system proposed is illustrated in simulation in section IV. Finally, section V draws the conclusions and describes some problems that warrant further research.

\section{INDIVIDUAL PATH FOLLOWING}

This section describes a sharped solution to the problem of path following control for one individual unicycle-type of mobile robot, where a helmsman-like behavior as a heading reference is injected. This meaningful behavior embedded in nonlinear controller design, is instrumental to render the error vector exponentially converging to zero.

The problem of path following is firstly addressed in [12] for kinematic model of wheeled mobile robot. Based on the Serret-Frenet frame $\{F\}$, tracking error vector between robot and the traced path is formulated. The origin of $\{F\}$ is the orthogonal projection of robot onto the path. However, it creates a singularity when the robot is located at the center of the path curvature such that the projection point is not uniquely defined. Consequently, only a local convergence of error vector is guaranteed. In order to bypass the singularity, a virtual target moving along the the path is introduced in [13]. Unfortunately, not only the speed of the virtual target but also that of robot have to be adjusted. In [14], the solution of global convergence is obtained by only steering the rotational angle, with the help of one extra control freedom to dexterously manipulate the speed of virtual target. Meanwhile, this method has been successfully extended from kinematics to dynamics. In this paper, this kind of individual path following design will be stretched, and an implicite helmsman-like behavior as a heading reference will be highlighted here.

\section{A. Kinematic and dynamic model}

Consider Figure 1, where a unicycle-type of wheeled robot follows a predefined spatial path. The robot has two identical parallel, non-deformable rear wheels and a passive front

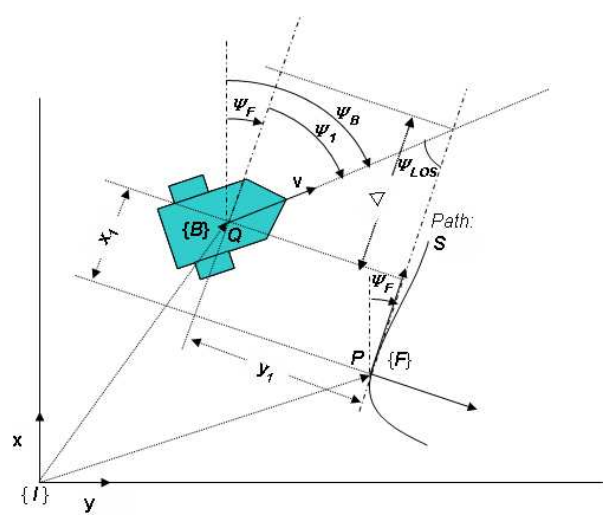

Fig. 1. Frame definition and description of problem posed

wheel. Let $v$ and $r$ denote the forward and rotational speed of the robot.

Let $P$ be an arbitrary point on the path to be followed and $Q$ be the center of mass of the moving robot. Associated with $P$, consider the corresponding Serret-Frenet frame $\{F\}$. The path $S$ is parameterized by a moving target $P$ on the path, with curvilinear abscissa (along path length) denoted by $s$. Let $\left(x_{1}, y_{1}\right)$ denote the coordinates of $Q$ in $\{F\}$. Let the rotations from $\{F\}$ to $\{I\}$ and $\{B\}$ to $\{I\}$ be denoted by the yaw angles $\psi_{F}$ and $\psi_{B}$, respectively. Further, let $c_{c}(s)$ and $g_{c}(s)$ denote the path curvature and its derivative respectively, and then $\psi_{F}=c_{c}(s) \dot{s}$. With the denotation of variable $\psi_{1}=\psi_{B}-\psi_{F}$, the kinematic model of unicycle in the Serret-Frenet frame can be derived as

$$
\left\{\begin{array}{l}
\dot{x}_{1}=-\dot{s}\left(1-c_{c} y_{1}\right)+v \cos \psi_{1} \\
\dot{y}_{1}=-c_{c} \dot{s} x_{1}+v \sin \psi_{1} \\
\dot{\psi}_{1}=r-c_{c} \dot{s}
\end{array}\right.
$$

where $r=\dot{\psi}_{B}$.

It is assumed the contact between the wheels of the robot and the ground is pure rolling and non-slipping. The wheels control provides the forward force $F$ and angular torque $N$ applied on the robot's center of mass. The robot mass and moment of inertia are denoted $m$ and $I$, respectively. The dynamical model of the unicycle is obtained by augmenting (1) with the equations

$$
\left\{\begin{array}{l}
\dot{v}=F / m \\
\dot{r}=N / I
\end{array}\right.
$$

Without loss of generality, $m=I=1$ is assumed in appropriate units.

With the above notation, the problem of path following for single robot can be formulated as below:

Individual Path Following. Given a predefined path to be followed by a individual robot, and given a desired speed profile $v_{d}(t) \geq v_{\text {min }}>0$ for the robot speed $v$, derive a feedback control law for $F$ and $N$ to drive $x_{1}, y_{1}, \psi_{1}$, and $v-v_{d}$ asymptotically to zero. 


\section{B. Nonlinear controller design}

The controller design for individual path following, is implemented in two steps.

(1) design a yaw angle as a heading reference, where a Line-of-sight (LOS) angle stands up, and a helmsman-like behavior is capsuled inside.

(2) design nonlinear controllers to drive the robot onto the path under the LOS guidance, which are based on Lyapunov theory and backstepping techniques.

1) Heading reference design: In order to follow the desired path, the most important thing is to steer the robot in the right heading to approach the objective, and the desired speed is of second interest. However, the performance of moving towards the path could be quite different, depending on the situation whether a reasonable heading is chosen and a wise computerized 'helmsman' is onboard .

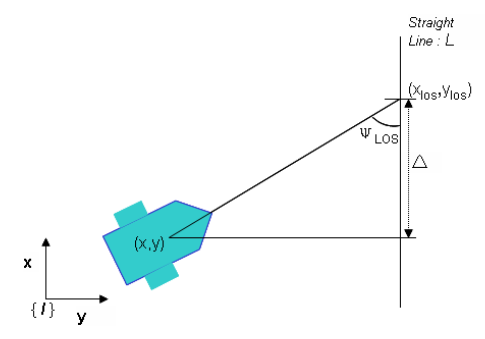

Fig. 2. Illustration of the LOS angle as a heading reference

Classic LOS law for heading reference is popularly applied in marine vehicles [15],[16], and this method enlightens us on designing the heading reference for wheeled robot here.

As depicted in figure 2, the coordinate origin of vehicle is $(x, y)$, and the LOS point on the path is $\left(x_{l o s}, y_{l o s}\right)$. Thus, the desired yaw angle under LOS guidance is

$$
\psi_{\text {los }}=\arctan \left(\frac{y_{\text {los }}-y}{\Delta}\right)
$$

Originally, the control parameter $\Delta(>0)$ is interpreted as the distance ahead of the ship along the $\mathrm{x}$-axis, i.e. look ahead distance towards the straight-line path.

Here we choose LOS angle as a heading reference, when vehicle tracking arbitrary path, and the parameter $\Delta$ is extended to look at the distance along the tangential path in Serret-Frenet frame.

Revisiting figure 1 , in the ideal case of $\psi_{1}$ equal to the desired heading $\delta$, we can see that $\psi_{1}=\psi_{B}-\psi_{F}$, is the corresponding LOS angle $\psi_{\text {los }}$ described in figure 2. That means the guidance yaw angle can be defined as

$$
\delta=\arctan \left(\frac{-y_{1}}{\Delta}\right)
$$

Where $\Delta$ can be given by

$$
\Delta=k_{0}\left|c_{c}(s)\right|+\epsilon, 0<k_{0}<k_{0 \max }, \epsilon>0
$$

In the situation of arbitrary path as in picture 1, the look ahead distance $\Delta$, manipulated by a wise helmsman, will steer the vehicle onto the tangential path. By commanding a large $\Delta$, the 'helmsman' gives a mild approach to the smooth curve, while a small $\Delta$ bringing more aggressive approach to the sharp curve. Explicitly, it is convenient for controller in the Serret-Frenet frame to provide the information of curvature, which means the helmsman behavior can be well embedded in the path following design proposed in this paper. Normally, $\Delta$ could be chosen equal to two vehicle's length, which is corresponding to a standard choice in LOS algorithms. Moreover, we can see later, that the LOS guidance is also instrumental in nonlinear controller design to sharpen the performance of convergence.

2) Kinematic controller: Consider the following Lyapunov function candidate

$$
V_{1}=\frac{x_{1}^{2}+y_{1}^{2}+\left(\psi_{1}-\delta\right)^{2}}{2}
$$

Resorting to the kinematics model (1), the derivative of $V_{1}$ is

$$
\begin{aligned}
\dot{V}_{1}= & x_{1}\left(v \cos \psi_{1}-\dot{s}\right)+y_{1} v \sin \delta+ \\
& \left(\psi_{1}-\delta\right)\left(\dot{\psi}_{1}-\dot{\delta}+y_{1} v \frac{\sin \psi_{1}-\sin \delta}{\psi_{1}-\delta}\right)
\end{aligned}
$$

It is straightforward to show that the choice

$$
\left\{\begin{array}{l}
\dot{s}=k_{1} x_{1}+v \cos \psi_{1} \\
\dot{\psi_{1}}=\dot{\delta}-y_{1} v \frac{\sin \psi_{1}-\sin \delta}{\psi_{1}-\delta}-k_{2}\left(\psi_{1}-\delta\right)
\end{array}\right.
$$

where $k_{1}$ and $k_{2}$ are positive gains, lead to

$$
\dot{V}_{1}=-k_{1} x_{1}^{2}+y_{1} v \sin \delta-k_{2}\left(\psi_{1}-\delta\right)^{2}
$$

With the heading reference designed in (3),

$$
\dot{V}_{1}=-k_{1} x_{1}^{2}-\frac{v y_{1}{ }^{2}}{\sqrt{y_{1}^{2}+\Delta^{2}}}-k_{2}\left(\psi_{1}-\delta\right)^{2}
$$

That means $\dot{V}_{1}<0$ outside the origin.

3) Dynamic controller: In the overall control loop, the kinematic controller actually acts as a reference subsystem, giving the desired signals for the control subsystem based on the dynamics level. Using backstepping techniques [17], the control law in kinematic level can be extended to deal with vehicle dynamics.

Let $\psi$ (desired value) be the reference signal of $\psi_{1}$ (actual value), which is derived from kinematic model. Let $\varepsilon$ be the error between $\dot{\psi}$ and $\dot{\psi}_{1}$. Then

$$
\left\{\begin{array}{l}
\dot{\psi}=\dot{\delta}-y_{1} v \frac{\sin \psi_{1}-\sin \delta}{\psi_{1}-\delta}-k_{2}\left(\psi_{1}-\delta\right) \\
\varepsilon=\dot{\psi}_{1}-\dot{\psi} \\
\dot{\varepsilon}=-\left(\psi_{1}-\delta\right)-k_{3} \varepsilon
\end{array}\right.
$$

With above equation, the control input of $N$ is

$$
\begin{aligned}
N= & \dot{r}=\ddot{\psi}_{1}+c_{c} \ddot{s}+g_{c} \dot{s}^{2} \\
& =\ddot{\psi}+c_{c} \ddot{s}+g_{c} \dot{s}^{2}-\left(\psi_{1}-\delta\right)-k_{3} \varepsilon
\end{aligned}
$$

Augment $V_{1}$ in Lyapunov function canditate $V_{2}$, then

$$
V_{2}=V_{1}+\frac{\varepsilon^{2}}{2}
$$

Substitute $\dot{\psi}_{1}$ with $\varepsilon+\dot{\psi}$ when computing $\dot{V}_{1}$, the derivative of $V_{2}$ is

$\dot{V}_{2}=-k_{1} x_{1}^{2}+y_{1} v \sin \delta-k_{2}\left(\psi_{1}-\delta\right)^{2}+\varepsilon\left(\dot{\varepsilon}+\left(\psi_{1}-\delta\right)\right)$ 
With (3) and (8), there is

$$
\dot{V}_{2}=-k_{1} x_{1}{ }^{2}-\frac{v y_{1}{ }^{2}}{\sqrt{y_{1}^{2}+\Delta^{2}}}-k_{2}\left(\psi_{1}-\delta\right)^{2}-k_{3} \varepsilon^{2}
$$

That means $\dot{V}_{2}<0$ outside the origin.

Proposition 1: Let guidance heading be given by (3), and control law be given by (9) for some $k_{i}>0(i=1,2,3)$. Let $\min \left\{k_{1}, k_{2}, k_{3}\right\} \geq \frac{v}{\Delta}, v \geq v_{\min }>0$ and $\Delta>0$ be guaranteed. Then the equilibrium point $\left(x_{1}, y_{1}, \psi_{1}\right)=$ $(0,0,0)$, is globally uniformly exponentially stable.

This proposition can be derived from (11), and there are two remarks here:

(1)Singularity is bypassed by the introduction of an extra degree of freedom for control design, which is attached to the virtual target. See [14] in detailed analysis.

(2)Actually, this is a sharped solution to the traditional path following control, both on mathematic level (performance of global convergence) and physic level (performance of helmsman-like behavior).

\section{CoOrdinated Path Following}

Before addressing this problem, the communication topology among the multi-robot system has to be explicitly represented. The algebraic graph theory is a useful tool, to derive decentralized controller for a team of robots.

\section{A. Preliminaries of algebraic graph theory}

We will review the basic concept of graph and matrices associated with graph, which are the preliminaries of algebraic graph theory and Laplacian matrix.

An communication topology is defined by a undirected graph $G(V, E)$ with $N$ vertices in a set of vertices $V$, and a set of edges $E$ with edges $e_{i j}=\left(v_{i}, v_{j}\right) \in E, v_{i}, v_{j} \in V$. Vertex $v_{i}$ and $v_{j}$ are connected if $\left(v_{i}, v_{j}\right) \in E$, and two vertices on the same edge are adjacent. The adjacent matrix $A$ of graph $G$, is a square matrix of size $|V|$, whose $i j$ th element $a_{i j}=1$ if $\left(v_{i}, v_{j}\right) \in E$, and is zero otherwise. The degree matrix $D$ of an undirected graph $G$, is the diagonal matrix with the number of its neighbors of each vertex along the diagonal denoted by $\operatorname{deg}\left(v_{i}\right)$, where the set of neighbors of node $i$ is denoted by $N_{i}=\left\{j:\left(v_{i}, v_{j}\right) \in E\right\}$. The Laplacian matrix $L$ of an undirected graph is defined as $L=$ $D-A$. A path is a sequence of edges from $v_{i}$ to $v_{j}$, such that two consecutive vertices are adjacent. A graph is said connected if there is a path between any distinct pair of vertices.

Lemma 1: From [18], the laplacian potential $L$ of a (connected) undirected graph is positive semi-define and satisfies the following identity, $S^{T} L S=\sum_{i, j \in E}\left(s_{i}-s_{j}\right)^{2}$, where $S=\left[s_{1}, s_{2}, \ldots, s_{n}\right]^{T}$ is the state vector of vertices. $s_{i}$ can be position, velocity, acceleration, etc.

Lemma 2: From [18], the Laplacian matrix of a connected graph, only has one single zero eigenvalue and the corresponding eigenvector is the vector of ones, $\overrightarrow{1}$.

\section{B. Coordinated controller design}

Deliberately, the design of control input $F$ in dynamics level, is lagged here. Consider Lyapunov function candidate, $V_{v}=\frac{1}{2}\left(v-v_{d}\right)^{2}$. It is trivial to choose the control law $\dot{v}=\dot{v}_{d}-k_{4}\left(v-v_{d}\right)$ where $k_{4}>0$, or rather that

$$
F=\dot{v}=\dot{v}_{d}-k_{4}\left(v-v_{d}\right)
$$

With (12), control force solely drives the robot speed $v$ converging to desired speed $v_{d}$ assuming $v_{d}(t) \geq v_{\min }>0$ , with performance of global uniform exponential stable. It indicates that controlling $v$ is completely decoupled with other control behaviors, such as, driving the robot onto the path with $x_{1}, y_{1}, \psi_{1}$ equal to zero. This important theoretic root endows the controller with another dedicated ability of speed adaptation among robots, without degrading the performance of robot's convergence to the path.

Therefore, the feasible strategy for coordinated path following is that

(1) each robot will recruit its own path following control law to track the path,

(2) and then, adjusting the desired speed of each robot, make the synchronizing parameters, tracked curvilinear abscissa (length along the path) $s_{i}(i=1,2, \cdots, n)$ herein, to be equal.

In this paper, it is assumed that, (1) the communications between any pair of robot is reciprocal (i.e. bidirectional link) and the graph is undirected, such that $L$ is symmetric, and (2) the communication graph is connected.

Coordinated Path Following. Consider $n$ robots with kinematic and dynamic models given by (1) and (2), respectively. Given $n$ spatial parallel paths to be followed by robots, and a desired profile $V_{d}$ for the final speed along the paths, derive feedback control laws, so that $x_{1, i}, y_{1, i}, \psi_{1, i}, v_{i}-v_{d_{i}}$, and $s_{i}-s_{j}(i=1,2, \ldots, n)$ tend to zero asymptotically.

The coordinated controller design for coordinated path following, is derived in four steps as following.

Step1: Given individual path following control law (9) for each robot, the multi-robot system uniformly globally exponentially reach the largest invariant set $\left\{\Omega_{P a t h} \mid\left(x_{1, i}, y_{1, i}\right)=\right.$ $\left.0^{2}, \psi_{i}=0, i=1,2, \cdots, n\right\}$

Step2: Given individual path following control law (12) for each robot, the multi-robot system uniformly globally exponentially reach the largest invariant set $\left\{\Omega_{v} \mid\left(x_{1, i}, y_{1, i}\right) \in\right.$ $\left.\Re^{2}, \psi_{i} \in \Re, v_{i}=v_{d i}, i=1, \cdots, n\right\}$

Step3: Let's study the trajectories of the robots onto the largest invariant set $\Omega_{P a t h}$ and $\Omega_{v}$. Under these two invariant sets, that is $\left\{\Omega_{P a t h} \cap \Omega_{v}\right\}$, all of robots are on their own paths and will move along these paths with desired speeds. That means, each robot coincides with the corresponding virtual target moving on the individual path. So, we can claim that $\dot{S}=V_{d}$ as along as the control laws exist, where the desired speed profile $V_{d}=\left[v_{d 1}, v_{d 2}, \ldots, v_{d n}\right]^{T}$, and $S=$ $\left[s_{1}, s_{2}, \ldots, s_{n}\right]^{T}$.

Considering Lyapunov candidate function $V_{S}=\frac{1}{2} S^{T} L S$, $V_{S}$ has a quadric form such that $V_{S} \geq 0$, as illustrated in Lemma 1. It is assumed there are reciprocal communication 
links among each pair of nodes, which contributes to symmetric Laplacian matrix $L=L^{T}$. Then, the time-derivative of $V_{S}$ is $\dot{V}_{S}=S^{T} L \dot{S}$.

Let the desired speed profile be

$$
V_{d}=\left\{v_{a} \overrightarrow{1}-v_{b} \tanh [L S]\right\}
$$

Note that:

(1) $[L S]=\left[L_{1} S, \ldots, L_{n} S\right]^{T}, \quad \tanh [L S]=$ $\left[\tanh \left(L_{1} S\right), \ldots, \tanh \left(L_{n} S\right)\right]^{T}$, and $L_{i}$ represents the $i$ th row of Laplacian matrix $L$.

(2) All robots have the same the minium and maxim speeds, noted as $v_{\min }$ and $v_{\max }$ respectively, and $v_{\max }>$ $v_{\min }>0$. Let $\left(v_{a}-v_{b}\right) \geq v_{\min }$ and $\left(v_{a}+v_{b}\right) \leq v_{\max }$, and $v_{a}>v_{b}>0$, to guarantee $v_{d i} \in\left[v_{\text {min }}, v_{\text {max }}\right]$ in (13).

Proposition 2: Consider the communication topology of multi-robot system represented by a connected graph with reciprocal links, let individual path following controller be given by (9) and (12). Let decentralized speed adaptation be given by (13). Then the multi-robot system globally asymptotically synchronized to an invariant manifold $\left\{\Omega_{S} \mid L S=\right.$ $0\}$, that is $s_{1}=s_{2}=\ldots=s_{n}$. Meanwhile, the speeds of all robots globally asymptotically convergence to a constant value $v_{a}(>0)$.

Proof: As the trajectories of the system asymptotically converge to the invariant set $\Omega_{\text {Path }}$ and $\Omega_{v}, \dot{S}$ equals to $V_{d}$.

$$
\dot{\mathbf{V}}_{\mathbf{S}}=v_{a} S^{T} L \overrightarrow{1}-v_{b}[L S]^{T} \tanh [L S]
$$

From Lemma $2, L \overrightarrow{1}=0 \cdot \tanh (x)$ is an odd function, $\left(L_{i} S\right) \tanh \left(L_{i} S\right) \geq 0$. In addition, $v_{b}>0$, such that $v_{b}[L S]^{T} \tanh [L S] \geq 0$. So, $\dot{\mathbf{V}}_{\mathbf{S}}=-v_{b}[L S]^{T} \tanh [L S] \leq 0$. That means, $V_{S}$ is a nonnegative and monotonically decreasing function, has a well-defined limit $\lim _{t \rightarrow \infty} V_{S}=l_{1}$, which means $V_{S}=S^{T} L S$ is bounded.

Moreover, it is straightforward to show that $\ddot{V}_{S}$ is bounded so that $\dot{V}_{S}$ is uniformly continuous. Then, using Barbalat's lemma, $\dot{V}_{S}$ tends to 0 as t tends to $\infty$, or rather that $\dot{\mathbf{V}}_{\mathbf{S}}=$ $-v_{b}[L S]^{T} \tanh [L S]$ tends to 0 . With $\left(L_{i} S\right) \tanh \left(L_{i} S\right) \geq 0$, $\left(L_{i} S\right) \tanh \left(L_{i} S\right)=0$, and $L_{i} S=0$ at last.

Now, we can conclude that the state of the system converges to the largest invariant subset, i.e. invariant manifold $M=\left\{S \in \Re^{n} \mid L S=0\right\}$, under decentralized control law of speed adaptation(13).

If the graph is connected, the invariant manifold $M$ implies that, $S$ are eigenvectors of $L$ corresponding to the single zero eigenvalue from Lemma 2. In another word, $S$ belong to span $\{\overrightarrow{1}\}$ and $M=\left\{S \in \Re^{n} \mid s_{1}=s_{2}=\ldots=s_{n}\right\}$.

Step4: We use LaSalle's invariance principle [19] to concatenate the two previous convergence properties, since our system can be considered as autonomous.

Let $\Omega=\Re^{2}$. The first and second step of the proof showed that every solution starting in $\Omega$ asymptotically converges to the invariant $\left\{\Omega_{\text {path }} \cap \Omega_{v}\right\}$. The third step showed that the largest invariant set of $\left\{\Omega_{\text {path }} \cap \Omega_{v}\right\}$, is the invariant manifold $M$. Therefore, every bounded solution starting in $\Omega$ converges to invariant manifold $M$ which indeed is $s_{1}=$ $s_{2}=\ldots=s_{n}$, as $t$ tends to $\infty$.
Consequently, $V_{d}=\left\{v_{a} \overrightarrow{1}-v_{b} \tanh [L S]\right\}=v_{a} \overrightarrow{1}$, which means each robot will always have the same velocity, to keep the same state value of $s_{i}$ upon synchronizing the state $S$, so the robots will be coordinated to follow the predefined paths in in-line formation.

\section{SIMULATIONS}

This section contains the results of simulation that illustrate the performance obtained with the control laws developed.

Four unicycle-type of wheeled mobile robots were required to follow four corresponding circumferences, which are with the same center but different radii $r_{i}(i=1,2,3,4)$, respectively, while keeping synchronization with in-line formation. That means the normalization of the along paths lengths $\hat{s}_{i}=s_{i} / r_{i}$ and normalization speed $\hat{v}_{i}=v_{i} / r_{i}$, will be the same. Actually, these normalized parameters make the truth, that the rotating speeds of virtual robots with respect to the same center of circles(i.e.anglar frequencies), as well as normalized lengths along paths, are synchronized. Therefore, the in-line formation of multi-robot system is built.

The radius of the circumferences are $r=[5,10,15,20] \mathrm{m}$. Four robots are with initial velocities of $v_{0}=[2,2,2,2] \mathrm{m} / \mathrm{s}$. The maxim and minium speed of the robots are, $v_{\max }^{d}=$ $6 \mathrm{~m} / \mathrm{s}$ and $v_{\min }^{d}=0.1 \mathrm{~m} / \mathrm{s}$. The initial positions are $x=$ $[0,0,0,0] m$ and $y=[0,-5,-10,-15] \mathrm{m}$. The initial tracing error vectors are $x_{1}=[5,5,5,5] \mathrm{m}$ and $y_{1}=[5,5,5,5] \mathrm{m}$. The initial error angles are $\psi_{1}=[p i / 4, p i / 4, p i / 4, p i / 4]$.

The Laplacian matrix, corresponding to the communication topology of the multi-robot system, is

$$
L=D-A=\left(\begin{array}{rrrr}
2 & -1 & 0 & -1 \\
-1 & 2 & -1 & 0 \\
0 & -1 & 2 & -1 \\
-1 & 0 & -1 & 2
\end{array}\right)
$$

As illustrated in figure 3, the along path lengths of different robots converge to the same normalized value. The speed converge to the desired speed profile $V_{d}=[1,2,3,4] \mathrm{m} / \mathrm{s}$, as illustrated in figure 4 . The normalized errors $s_{i j}\left(=\hat{s}_{i}-\hat{s}_{j}\right)$ and $v_{i j}\left(=\hat{v}_{i}-\hat{v}_{j}\right)$ are illustrated in figure 5 and 6 , decaying to 0 respectively.

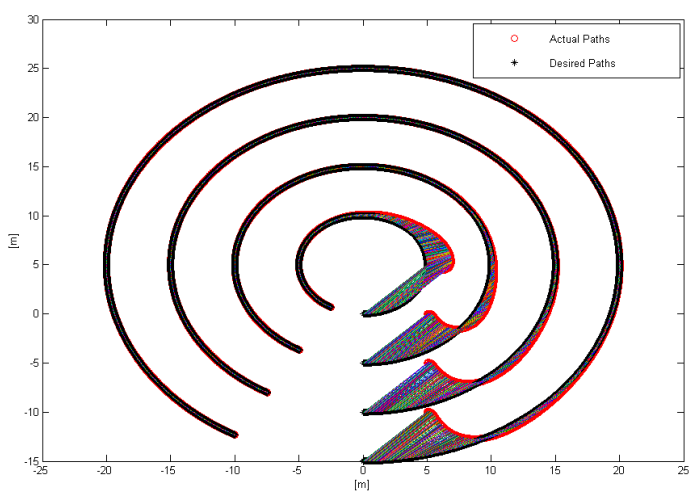

Fig. 3. Coordinated path following in in-line formation. 


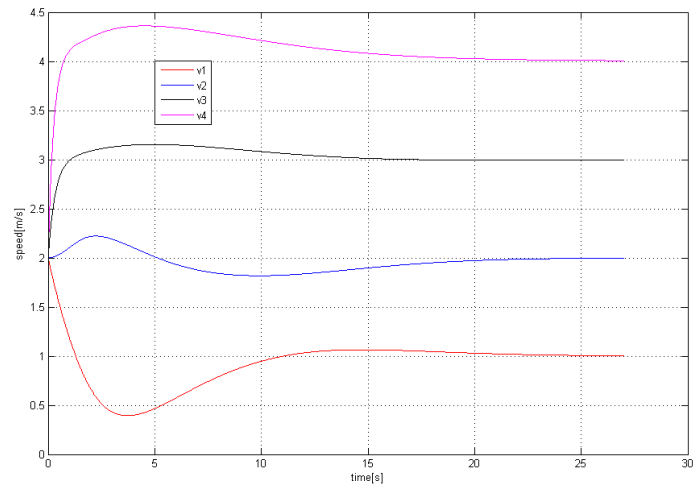

Fig. 4. Coordinated speeds in in-line path following.

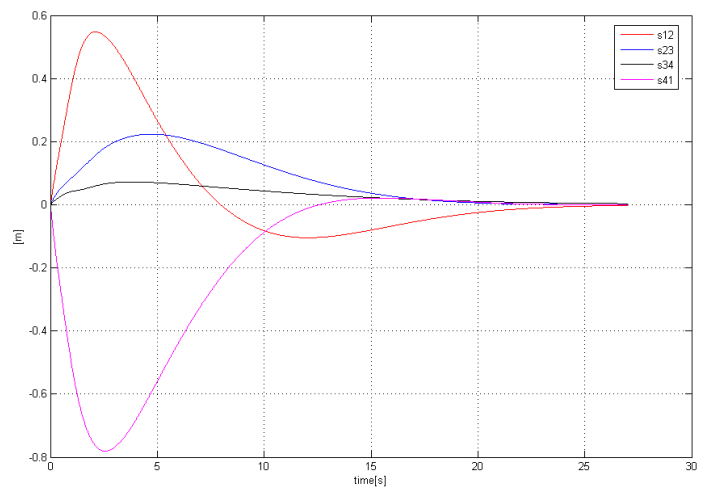

Fig. 5. Normalized along path errors in in-line path following.

\section{CONCLUSIONS AND FUTURE WORKS}

The paper addressed the problem of coordinated path following of multiple robots. There are two-layer controllers for each robot, decoupled in geometric task and dynamic speed adapting task. One is the individual path following controller, which drives the robot converging to the paths, with a helmsman-like behavior embedded in heading reference design. The other is the controller for coordination in global sense, which is realized by means of decentralized speed adaptation. The minium communication variables is requested here, and the communication topology is not necessarily all-to-all. A formal proof of convergence for each (individual/coordinated path following) controller is derived in detail, and simulations illustrated the efficacy of the solution proposed. Further work will address the case where multiple robots are required to following generalized spatial paths. Switching topology and time delays inside the communication network are also of interest to be integrated into coordinated path following.

\section{REFERENCES}

[1] R.Beard, J.Lawton, and F.Hadaegh. A coordination architecture for spacecraft formation control. IEEE Trans. Contr. Syst. Tech., vol. 9, 1999, pp $777-790$.

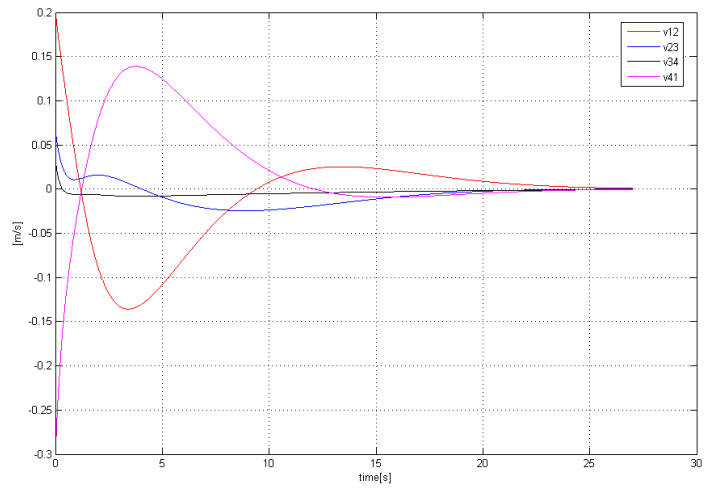

Fig. 6. Normalized speed errors in in-line path following.

[2] J.Desai, J.Otrowski, and V.Kumar. Controlling formations of multiple robots. IEEE International Conference on Robotics and Automation (ICRA98), 1998, pp 2864-2869.

[3] L. Alvarez and R. Horowitz. Traffic Flow Control In Automated Highway Systems. Research Reports: Paper UCB-ITS-PRR-97-47, California Partners for Advanced Transit and Highways (PATH), 1997

[4] F. Zhang, D. M. Fratantoni, D. Paley, J. Lund, and N. E. Leonard, Control of coordinated patterns for ocean sampling, International Journal of Control, 2007, pp. 1186-1199.

[5] P.Encarnacao, and A.Pascoal, 3D path following for autonomous underwater vehicle, Proceedings of CDC 2000, 39th IEEE Conference on Decision and Control, Sydney, Australia. 2000.

[6] L.Lapierre, D.Soetanto, and A.Pascoal, Coordinated Motion Control of marine robots, Proc. MCMC 2003 - 6th IFAC Conference on Manoeuvering and Control of Marine Craft, Girona, Spain, 2003

[7] I.Kaminer, O.Yakimenko, A.Pascoal, Ghabcheloo, R. Path Generation, Path Following and Coordinated Control for Time Critical Missions of Multiple UAVs. IEEE American Control Conference, volume, Issue , 14-16 June 2006, pp 4906 - 491

[8] A. Fax and R. Murray, Graph Laplacians and stabilization of vehicle formations, IEEE Transactions on Automatic Control, vol. 49, 2004, pp 1465-1476

[9] R. O. Saber and R. M. Murray, Consensus Problems in Networks of Agents with Switching Topology and Time-Delays, IEEE Trans. on Automatic Control, 2004, vol. 49(9), pp. 1520-1533.

[10] R. Ghabcheloo, A. Pascoal, C. Silvestre, and I. Kaminer, Nonlinear coordinated path- following control of multiple wheeled robots with bi-directional communication constraints, International Journal of Adaptive control and signal Processing, vol. 21, 2007, pp. 133-157.

[11] R. Skjetne, S. Moi, and T. Fossen, Nonlinear formation control of marine craft, in Conference on Decision and Control, Las Vegas, NV., 2002, pp. 680-685.

[12] C. Samson, Path following and time-varying feedback stabilization of a wheeled mobile robot, Proceedings of the International Conference on Advanced Robotics and Computer Vision, Singapore, vol.13, 1992, pp. 1.1-1.5.

[13] M. Egerstedt, X.Hu, and A.Stotsky, Control of Mobile Platforms Using a Virtual Vehicle Approach, IEEE Transactionon Automatic Control, vol. 46, 2001, pp.1777-1782.

[14] D. Soetanto, L. Lapierre, and A. Pascoal, Adaptive, non-singular path following control of a wheeled robot, in Proc. IEEE Conf. Decision Control, Maui, HI, Dec. 9-12, 2003, pp. 1765-1770.

[15] T. Fossen, Guidance and Control of Ocean Vehicles, Wiley, New York: 1994.8

[16] T. I. Fossen, M. Breivik, and R. Skjeme, Line-of-Sight Path Following of Underactuated Marine Craft, Proceedings of the 6th IFAC MCMC, Girona, Spain, 2003. pp.244-249.

[17] M. Krstic, I.Kanellakopoulos, and P.Kokotovic Nonlinear and Adaptive Control Design, John Willey \& Sons, Inc., New York:, 1995.

[18] C. Godsil and G. Royle, Algebraic Graph Theory, Graduated Texts in Mathematics, Springer- Verlag NewYork, Inc.; 2001.

[19] H. K. Khalil, Nonlinear Systems, Prentice-Hall, Upper Saddle River, NJ: 1996. 